\title{
Switch-like surface binding of competing multivalent particles ${ }^{*, * *}$
}

\author{
N.B. Tito ${ }^{\mathrm{a}}$ and D. Frenkel \\ Department of Chemistry, University of Cambridge, Lensfield Road, Cambridge CB2 1EW, \\ UK
}

Received 15 April 2016 / Received in final form 8 June 2016

Published online 18 July 2016

\begin{abstract}
Multivalent particles competing for binding on the same surface can exhibit switch-like behaviour, depending on the concentration of receptors on the surface. When the receptor concentration is low, energy dominates the free energy of binding, and particles having a small number of strongly-binding ligands preferentially bind to the surface. At higher receptor concentrations, multivalent effects become significant, and entropy dominates the binding free energy; particles having many weakly-binding ligands preferentially bind to the surface. Between these two regimes there is a "switch-point", at which the surface binds the two species of particles equally strongly. We demonstrate that a simple theory can account for this switch-like behaviour and present numerical calculations that support the theoretical predictions. We argue that binding selectivity based on receptor density, rather than identity, may have practical applications.
\end{abstract}

\section{Introduction}

Systems comprising many different chemical ingredients may exhibit complex physical behaviour that is absent in systems with fewer components. In fact, in many biological or bio-mimetic systems, variations in chemical composition allow the system to switch from one mode of operation to another. To give a specific example: living cells are sensitive to the nature of the molecules bound to their surface, but what molecules bind to a cell surface depends on the chemical composition of that surface [1]. A non-biological example is the self-assembly of an elaborate structure from many unique pieces of deoxyribonucleic acid (DNA) [2-6]: here the chemical composition of the system determines the shape of the units that form through self-assembly.

"Multivalent" particles are a broad class of supra-molecular structures that interact through multiple ligands with (multiple) chemically complementary targets ("receptors"). While the individual ligand-receptor bonds are typically weak, the

\footnotetext{
* Supplementary material in the form of one pdf file available from the journal web page at http://dx.doi.org/10.1140/epjst/e2016-60119-6

** The underlying data for this publication can be freely downloaded and viewed at http://dx.doi.org/10.17863/CAM.582

a e-mail: nicholas.b.tito@gmail.com
} 
multiplicity of possible host-guest binding combinations causes the overall binding strength of the particle to be extremely sensitive to system conditions, such as temperature, $\mathrm{pH}$, the number of ligands on the particle, and the receptor concentration on the targeted surface. The binding is superselective when the number of adsorbed particles increases faster than linearly with the concentration of receptors [7].

For a monovalent particle, binding is controlled by the strength of only one ligandreceptor bond. In that case, the number of adsorbed particles depends at most linearly on the surface density of available receptors. In contrast, for multivalent particles, the number of adsorbed particles may depend faster than linear on the surface density of available receptors. However, provided that the binding strength per ligand-receptor pair is small enough, the multivalent binding to a surface can still be reversible.

The physical origin of the high sensitivity of multivalent binding to the receptor density is entropic. Typically, unless all bonds are saturated (which only happens for strong binding) there are many ways in which a subset of all ligands can bind to a subset of all receptors. The (logarithm of) the number of ways in which these bonds can be made is an entropic factor that, typically, depends faster than linearly on the receptor concentration.

Biological systems often exploit multivalency to make bonding of a particle to a target surface extremely sensitive to changes in local environment [1]. The same holds for man-made multivalent interactions [8].

DNA-coated colloids are an example of synthetic, multivalent building blocks [9-13] that can self-assemble. In these systems, complementary single-stranded DNA is grafted to the surfaces of colloids or nanoparticles, resulting in a system that selfassembles over a narrow temperature range into aggregate structures "encoded" by the DNA ligands. A recent experimental study has demonstrated size-selective surface binding and patterning from a bimodal mixture of DNA-coated nanoparticles, depending on the local density of receptor DNA strands on the surface [14].

Because of their sensitivity to the nature and surface-concentration of receptors, multivalent particles are well suited for chemical and biological sensing [15]. In some cases, the sensing works by selective break-up of clusters. For instance, gold nanoparticles decorated with bio-responsive ligands have been used as sensing agents to detect the presence of complementary enzymes [16]. In the absence of these enzymes, the nano-particles form clusters via multivalent interactions between their ligands; however, this interaction is disrupted by the presence of the enzyme. As a result, the size of the nano-particle clusters (and hence their plasmon resonance) depends sensitively on the enzyme concentration.

In the present paper we show that, depending on their surface concentration, one and the same type of receptor can induce the binding of different multivalent particles. Varying the surface concentration of receptors acts as a "switch" between binding of two (or, at least in principle, more) types of multivalent particles.

To illustrate how such a switch works, we consider a flat surface covered with varying amounts of receptor molecules, in contact with a reservoir containing two distinct multivalent species. The multivalent particles are assumed to be spherical and coated with mobile flexible ligands; however, qualitatively, our conclusions depend neither on the shape of the particles, nor on the mobility of the ligands.

The outline of the remainder of this paper is as follows. We first develop a simple theory to predict the competitive adsorption of two multivalent species by estimating the equilibrium free energy of binding. Following that, we more accurately estimate the free energy of binding with a lattice model, accounting for the change in conformational entropy of a ligand when it binds to a receptor. In the third section we discuss the results obtained from the lattice model, illustrating the influence of energy and entropy on multivalent particle binding, and comparing our results with the relevant theoretical predictions. The paper concludes by discussing possible applications of multivalent switches. 


\section{Theory}

The competition for surface adsorption between two multivalent species can be illustrated by considering a simple Langmuir adsorption model [7]. In order to develop a mathematically tractable model, we make the following assumptions: the two multivalent particles have approximately equal sizes, and equal ligand lengths; the number of receptors available for binding when a particle is adjacent to the surface is much larger than the number of ligands on the particle; and the binding strength of both particle species is moderate to strong. We will relax these assumptions when examining detailed lattice model calculations in subsequent sections.

Consider a system containing a surface covered by randomly-placed receptors. A reservoir of two multivalent species is in contact with the surface; the two species are identified by $i$ and $j$. A particle of species $i$ has $N_{L, i}$ ligands, and the free energy of binding a single ligand to a receptor is $\Delta f_{i}$. Species $j$ similarly has parameters $N_{L, j}$ and $\Delta f_{j}$. When a particle belonging to either of the two species is adjacent to the surface, its ligands are able to access $N_{R}$ receptors in its vicinity.

The strength of the surface binding of either species depends on the value of $N_{L}$, $\Delta f, N_{R}$ and on the temperature $T$. All other things being equal, the species that has the lower free energy of surface binding will preferentially bind to the surface. Thus, by changing $\left(N_{L}, \Delta f\right)$ for the two species, we may introduce a competition for surface occupancy, and fine-tune which species binds for a given choice of global conditions $\left(T, N_{R}\right)$.

Following ref. [7], we can estimate the multivalent binding free energy as

$$
\begin{aligned}
\beta F_{b} & \approx N_{L}\left(\beta \Delta f-\ln N_{R}\right) \\
& =-N_{L} \ln N_{R}+\text { const }
\end{aligned}
$$

where $\beta=1 / k_{B} T$ and $k_{B}$ is the Boltzmann constant. In Eq. (1), we see that the free energy is proportional to $\ln N_{R}$, with a scaling factor of $N_{L}$. The binding free energies of species $i$ and $j$ are equal, i.e. $F_{b, i}=F_{b, j}$, when,

$$
\left(k_{B} T \ln N_{R}\right)_{\text {switch }}=\frac{N_{L, j} \Delta f_{j}-N_{L, i} \Delta f_{i}}{N_{L, j}-N_{L, i}} .
$$

The relation given by Eq. (2) defines a unique choice of the global parameters $\left(T, N_{R}\right)$ at which the two multivalent species bind equally strongly to the surface. In what follows, we will assume that the is temperature fixed: we will only vary the receptor count $N_{R}$.

Let us now consider the case that species $i$ has a small number of strongly binding ligands, while species $j$ has many less-strongly-binding ligands;

$$
\begin{aligned}
& N_{L, j} \gg N_{L, i} \\
& \Delta f_{i} \ll \Delta f_{j} .
\end{aligned}
$$

In this regime, we can still use Eq. (1) to predict how the difference in binding free energies between the two species depends on $N_{R}$ :

$$
\beta \Delta F_{j i, b} \approx \beta\left(N_{L, j} \Delta f_{j}-N_{L, i} \Delta f_{i}\right)-N_{L, j} \ln N_{R}
$$

We note that the binding free energy of species $i$, with a small number of strongly binding ligands, has a weak dependence on $N_{R}$ in this limit. However, the binding free energy of species $j$, with many weaker binding ligands, depends strongly on $\ln N_{R}$. When $N_{R}$ is large, then $F_{b, j}>F_{b, i}$, as the entropy of binding is the dominant factor in the bound-state free energy of the two species. On the other hand, when $N_{R}$ is 
small, then $F_{b, i}>F_{b, j}$, and the energy of binding determines which species binds to the surface. When $N_{R}$ satisfies Eq. (2), then the entropy and energy of binding of each species are at a balance.

The simple theory here reveals that switch-like behaviour occurs when the binding free energy for each species depends differently on the surface-receptor concentration. Therefore, mixtures of monovalent species will never exhibit surface switching. For example, if a system contains two monovalent species, one strongly binding, and the other weakly binding, then the ratio of the surface concentrations of the two species will not depend on the receptor concentration.

\section{Lattice model for ligand-receptor binding}

In the previous section, a key parameter was $\Delta f$, the binding free energy per ligand. This free energy has two contributions: the binding energy of the ligand-receptor interaction and the difference in configurational entropy of the ligand in the bound and unbound states. This configurational entropy depends on the nature of the ligand and on the space accessible to the ligand in its bound and unbound states.

To compute the approximate entropy change of a ligand upon binding, we use a lattice model. The model represents a single multivalent particle with ligands, interacting with receptors placed on a surface. We account for the fact that the ligands cannot penetrate the surface and that no two ligands can be bound to the same receptor. In Ref. [17] we describe an efficient method to compute the binding free energy of a multivalent particle, taking into account the above constraints.

We represent the system on a three-dimensional simple cubic lattice. A flat impenetrable surface is located at vertical coordinate $h=1$ (in lattice units), on which receptor sites are randomly placed. The core of the multivalent particle is represented on the lattice by a (discretised) hard sphere, with radius $r$, and center located at $\left(x^{*}, y^{*}, h^{*}\right)$. Impenetrability is enforced by preventing any ligand segments from occupying lattice sites with coordinates $(x, y, h)$ satisfying $\left(x-x^{*}\right)^{2}+\left(y-y^{*}\right)^{2}+\left(h-h^{*}\right)^{2} \leq r^{2}$. Given that the receptor surface is located at $h=1$, the distance between the receptor surface and particle surface is $z=h^{*}-r-2$.

The ligands of the multivalent particle are represented as non-self-avoiding lattice walks of $N_{\text {poly }}$ steps. Recursive enumeration [18] is used to calculate the number $q_{j}^{\prime}$ of walks that extend from anywhere on the particle core surface, to site $j$ on the receptor surface. The energy of a single ligand-receptor bond is $\epsilon$ for all receptors; the total binding weight for site $j$ is therefore $q_{j}=q_{j}^{\prime} \exp (-\beta \epsilon)$. Each of the $N_{L}$ ligands belonging to the particle are assumed to be chemically identical, so that there is no dependence on ligand identity when computing the binding weights $q_{j}$. The partition function $q_{u b}$ per unbound ligand is defined to be the number of non-self-avoiding walks beginning on the particle surface and ending anywhere in the system. Each $q_{j}$ as well as $q_{u b}$ depend on the spacing $z$ between the particle and receptor surfaces.

We denote by $N_{A}(z)$ the number of surface sites accessible to the particle ligands, when the particle has spacing $z$ from the receptors surface. $N_{A}(z)$ is determined by geometry: for a particle having ligands of length $N_{\text {poly }}$, at a distance $z$ from the surface $N_{A}(z) \approx 2\left(N_{\text {poly }}-z+1\right)^{2}$ and the mean number of receptors available to the particle is

$$
\bar{N}_{R}(z)=N_{A}(z) \phi_{R},
$$

where $\phi_{R}$ is the probability to find a receptor at a surface site.

To calculate the binding free energy, we must average over all possible surface receptor configurations. We assume that receptors are randomly distributed over the $N_{A}(z)$ accessible surface sites. The probability $P\left(N_{R}\right)$ to find exactly $N_{R}$ receptors 
on $N_{A}(z)$ sites is then:

$$
P\left(N_{R}\right)=\left(\begin{array}{c}
N_{A} \\
N_{R}
\end{array}\right) \phi_{R}^{N_{R}}\left(1-\phi_{R}\right)^{N_{A}-N_{R}},
$$

where we have written $N_{A}$ for $N_{A}(z)$. From ref. [17], the bound-state partition function for $z$ averaged over every possible receptor configuration is

$$
Q_{\text {full }}=\sum_{\lambda=0}^{\min \left(N_{A}, N_{L}\right)} Q(\lambda),
$$

where

$$
Q(\lambda)=\frac{\left(\begin{array}{c}
N_{L} \\
\lambda
\end{array}\right)}{\left(\begin{array}{c}
N_{A} \\
\lambda
\end{array}\right)} \lambda ! Q_{u b}(\lambda) Q_{b}\left(N_{A}, \lambda\right) \times \sum_{N_{R}=\lambda}^{N_{A}}\left(\begin{array}{c}
N_{R} \\
\lambda
\end{array}\right) P\left(N_{R}\right) .
$$

The quantity $Q_{b}\left(N_{A}, \lambda\right)$ is computed by finding the residue of a complex integral [17]. The quantity $Q_{u b}(\lambda)=q_{u b}^{\left(N_{L}-\lambda\right)}$ is the partition function for the unbound ligands. The bound free energy is then obtained by

$$
F(z)=-k_{B} T \ln Q_{f u l l} .
$$

It is useful to calculate this quantity relative to the free energy $F^{\circ}$ when the particle is at infinite distance from the surface, where $F^{\circ}=-N_{L} \ln q^{\circ}$ and $q^{\circ}$ is the partition function for one ligand in the reference state. The free energy of binding is therefore

$$
\Delta F(z)=F(z)-F^{\circ} .
$$

Equation (5) is calculated for different values of $z$. The position $z^{*}$ that leads to the most negative binding free energy $\Delta F^{*}$ is taken to be the equilibrium position for fixed $\left(N_{\text {poly }}, N_{L}, \beta \epsilon, \phi_{R}\right)$.

\section{Results \& discussion}

We now numerically calculate the free energy of binding for surface-adsorbing multivalent particles, as a function of the concentration of surface receptors. Competition between two particle species is illustrated by comparing their binding free energy to the same surface. In the Supporting Information, we perform a more detailed analysis of how multivalency affects the entropy and energy of binding, including the limit of strong-binding ligands.

Two species of particles are defined in the system: one with few strong-binding ligands, and the other with many weak-binding ones. The first species, $i$, has $N_{L, i}=1$ and $\epsilon_{i}=-12 k_{B} T$, while the second species $j$ has $N_{L, j}=20$ and $\epsilon_{j}=-3.5 k_{B} T$. While quantitatively, our results depend on the parameters chosen, the broader concept of switch-like competition between the two species does not.

The results in Fig. 1 show the free energy of binding as a function of distance $z$ between the particle and receptor surface. Results are shown for different choices of receptor concentration $\phi_{R}$ at fixed temperature.

Figure 1(a) indicates that the low-valence particle with one strong-binding ligand has binding free energy minima very near the receptor surface. The value of the binding free energy at the minimum changes little with receptor concentration. On the 


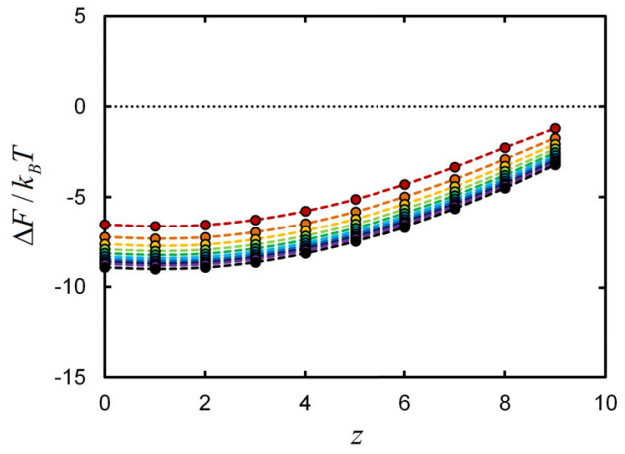

(a)

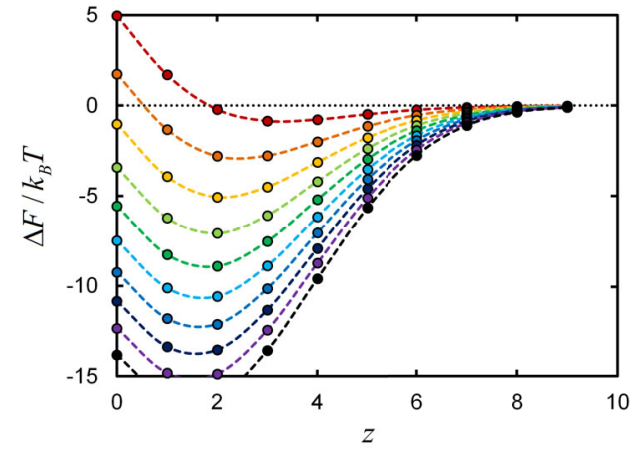

(b)

Fig. 1. Binding free energy $\Delta F / k_{B} T$ as a function of distance $z$ between receptor and particle surfaces, for different choices of receptor concentration $\phi_{R}$. Values of receptor concentration increase from red to dark violet, progressing 0.1 to 1.0 in increments of 0.1 . Points are numerical results, and lines are guides to the eye. In (a), $N_{L}=1$ and $\epsilon=-12 k_{B} T$; in (b), $N_{L}=20$ and $\epsilon=-3.5 k_{B} T$. Both particles have ligands of $N_{\text {poly }}=20$ segments, and cores of radius $r=2$.

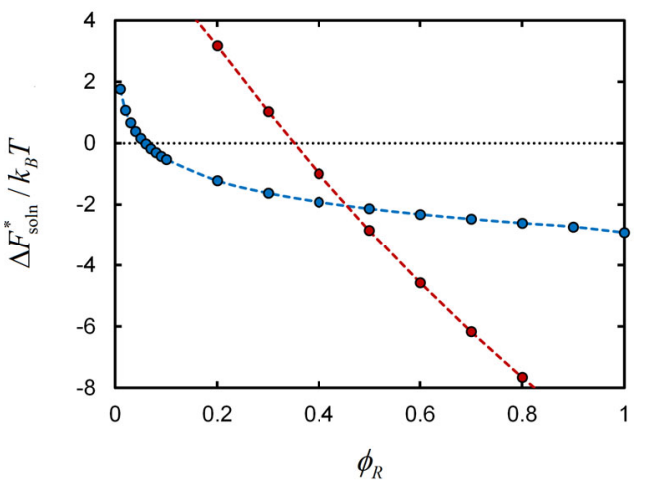

Fig. 2. Solution binding free energy $\Delta F_{\text {soln }}^{*} / k_{B} T$ vs. surface receptor concentration $\phi_{R}$ for the two particle species $i$ (blue) and $j$ (red). Solution chemical potential is $\beta \mu_{i d}=-6.1$. Points are numerical calculations, and lines are guides to the eye.

other hand, in Fig. 1(b), the particle with many weak-binding ligands has a preferred binding position some distance away from the surface, due to the effective repulsion caused by unbound ligands. With increasing receptor concentration the minimum in the free energy curve shifts closer to the surface. Moreover, the minimum in the binding free energy becomes deeper as $\phi_{R}$ increases.

We now consider the situation where the bulk concentration of each species is $\approx 0.002$ particles per unit volume, leading to an (ideal) chemical potential of $\beta \mu_{i d}=-6.1$. The free energy of binding of a particle from this hypothetical solution is calculated by

$$
\Delta F_{\text {soln }}^{*}=\Delta F^{*}-\mu_{i d},
$$

which incorporates the solution chemical potential, and the minimum free energies of binding from Fig. 1.

In Fig. 2, solution free energies of binding $\Delta F_{\text {soln }}^{*}$ are plotted as a function of $\phi_{R}$. At low receptor concentration, the low-valence species $i$ has a more negative (more favourable) binding free energy compared to the high-valence species $j$. As the 


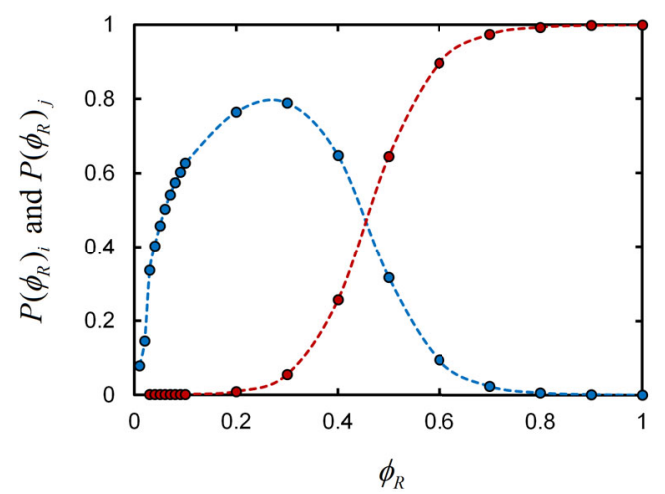

Fig. 3. Probability that species $i$ (blue) and $j$ (red) are bound to the surface as a function of receptor concentration. Points are numerical results, and lines are guides to the eye.

receptor concentration grows larger, the binding free energy of the high-valence species becomes more favourable. More receptors result in more possible ligand-receptor binding permutations for species $j$, causing the entropic contributon to the binding free energy for that species to become more significant. Near a receptor concentration of 0.5 , the two curves cross at a "switch point", and at larger $\phi_{R}$ the high-valence species is more favourably bound to the surface.

Figure 3 illustrates the switch point of this system in terms of the probability that a particular species is bound to the surface. For species $i$ for example,

$$
P\left(\phi_{R}\right)_{i}=\frac{e^{-\beta \Delta F_{\text {soln }, i}^{*}\left(\phi_{R}\right)}}{1+e^{-\beta \Delta F_{\text {soln }, i}^{*}\left(\phi_{R}\right)}+e^{-\beta \Delta F_{\text {soln }, j}^{*}\left(\phi_{R}\right)}} .
$$

where $\Delta F_{\text {soln }, i}^{*}\left(\phi_{R}\right)$ is the solution binding free energy for species $i$ at a receptor concentration of $\phi_{R}$. At low receptor concentration, species $i$ is bound weakly, consistent with its rather small binding free energy of $\approx-1 k_{B} T$. At larger receptor concentration, species $i$ binds more strongly initially, but then is replaced by the more favorably-bound species $j$.

Multivalent particles interact with receptors on a surface by different ligandreceptor pair combinations. This is like connecting the sockets of an old telephone switchboard in different ways. In the Supporting Information, we isolate and examine the role of "switchboard entropy" - the entropy associated with different ligandreceptor combinations - in the context of switch-like particle binding competition as seen in Fig. 2.

We next consider how the switch point of two competing multivalent species changes as a function of system parameters, such as valency $N_{L}$ and binding strength $\epsilon$. Equation (2) represents a rough prediction for the $N_{R}$ at which there is coexistence between two species $i$ and $j$. Equation (2) can be rearranged, separating out the energetic and entropic contributions, to yield

$$
\begin{aligned}
\ln N_{R, \text { switch }} & =\frac{\beta\left(N_{L, j} \epsilon_{j}-N_{L, i} \epsilon_{i}\right)}{N_{L, j}-N_{L, i}}-\frac{N_{L, j} s_{j} / k_{B}-N_{L, i} s_{i} / k_{B}}{N_{L, j}-N_{L, i}} \\
& =\eta-b .
\end{aligned}
$$

Represented in this way, the theory predicts that the switch-point $\ln N_{R}$ values will be directly proportional to $\eta$ with slope unity, and with an intercept of $b$ representing a binding entropy difference between the two species. The quantity $\eta$ is useful, as it 




Fig. 4. Switchpoint $\left(\ln \phi_{R}\right)$ as a function of binding parameter $\eta$ for competing multivalent surface adsorbers. Points are numerical calculations for a variety of $\eta$; solid line has slope of 1, indicating expected scaling between $\eta$ and $\ln \phi_{R}$ given by Eq. (6).

is a function only of the parameters used to define the two multivalent species in the system.

We now show that the switch-point receptor concentration $\phi_{R}$ scales linearly with the parameter $\eta$ as predicted by Eq. (6). Figure 4 shows values of $\left(\ln \phi_{R}\right)$ at which surface switching occurs for a variety of pairs of competing multivalent species $i$ and $j$. Choosing $\left(N_{L, i}, \epsilon_{i}\right)$ and $\left(N_{L, j}, \epsilon_{j}\right)$ for species $i$ and $j$ respectively leads to a unique value of the parameter $\eta$, as displayed in Eq. (6).

The results in Fig. 4 suggest that $\left(\ln \phi_{R, \text { switch }}\right)$ scales approximately linearly with $\eta$, as predicted by the simple theory in Eq. (6). At larger (more negative) $\eta$, the numerical results deviate from the theory. According to Eq. (6), a more negative $\eta$ leads to a smaller value of the switch-point receptor concentration $\phi_{R}$. Thus, we reasonably expect Eq. (6) to break down in this limit, given that large $N_{R}$ was assumed in the derivation in Sect. 2. Nevertheless, the agreement between the numerical calculations and the theory is surprisingly good, given that the theory also does not account for the polydispersity $P\left(N_{R}\right)$ of the local number of surface receptors.

\section{Conclusions}

The results presented in this paper indicate that the preferred adsorption of particles can be switched by changing surface receptor concentration, provided that one particle has few strong-binding ligands, while the second has many weak-binding ligands. Importantly, this behaviour is unique to multivalent particles, given that a mixture of monovalent species always exhibits the same coexistence ratio on the surface regardless of receptor concentration.

A lattice model coupled with a recent multivalent binding theory [17] was employed to develop a more detailed picture of switch-like adsorption. At low receptor concentration, the low-valence species having strong-binding ligands preferentially adsorbs to the surface. At larger receptor concentration, binding entropy associated with multivalency becomes a significant factor in the free energy, causing the high-valence species to occupy the surface instead. In between, there is a unique choice of receptor concentration at which the two species coexist. The coexistence points closely scale with a simple Langmuir theory for the cases where the theory assumptions are valid.

Examining the energy and entropy of binding in detail (see Supporting Information) reveals the role that receptor degeneracy plays in the binding process. The bound-state entropy of the multivalent species initially decreases as the number of 
receptors increases. This is due to the entropic cost associated with binding ligands to the surface. Once the particle has all of its ligands bound, there is a turning point, and the equilibrium entropy begins to increase again due to the availability of excess receptors.

This "switchboard" entropy dominates the free energy of binding of multivalent species when there are excess receptors, while having an insignificant role for monovalent species. Altering the number of ligands on a particle therefore tunes the magnitude of the switchboard entropy term in the binding free energy, and this can be used to drive competitive surface adsorption in a mixture of species.

Our present approach does not offer insight into the effect of kinetics on the switching rate of multivalent binding. However, there is no doubt that kinetics will play a role in any practical implementation.

Most importantly, the number and strength of the ligand-receptor bonds will affect the kinetics of binding. Strong ligand-receptor bonds have long lifetimes. When individual bonds are strong, the rate at which a multivalent particle can sample the surface concentration of receptors is decreased $[19,20]$. This potential problem can be alleviated by making the energy of ligand-receptor bonds weaker: doing so would not be at the expense of a high multivalent binding selectivity [7].

The multivalent physics discussed in this paper suggest development of a type of "multivalent chromatography", in which a sensing surface is used to isolate particles from a polydisperse mixture based on their valency. Recent experimental progress on the development of self-assembled monolayers having chemical gradients is promising [21], as these would serve as ideal substrates for performing the chromatography. DNA dendrimers [22] present an opportunity to experimentally study surface switching behaviour in a controlled fashion, given the ability to synthesize dendrimers with specific valency and ligand interaction energy. These applications will be the subject of forthcoming work.

The research leading to these results has received funding from the European Research Council under the European Union's Seventh Framework Programme (FP/2007-2013) / ERC Grant Agreement n. 607602 ("SASSYPOL"). Funding is also gratefully acknowledged from EPSRC Programme Grant EP/I001352/1. We wish to thank Tine Curk, Stefano AngiolettiUberti, and Peter Bolhuis for helpful discussions on this work. DF gratefully acknowledges his many interactions with Kurt Kremer, spanning almost 3 decades.

\section{References}

1. M. Mammen, S.K. Choi, G.M. Whitesides, Angew. Chem. Inter. Ed. 37, 2754 (1998)

2. Y. Ke, L.L. Ong, W.M. Shih, P. Yin, Science 338, 1177 (2012)

3. J.D. Halverson, A.V. Tkachenko, Phys. Rev. E 87, 062310 (2013)

4. A. Reinhardt, D. Frenkel, Phys. Rev. Lett. 112, 238103 (2014)

5. L.O. Hedges, R.V. Mannige, S. Whitelam, Soft Matter 10, 6404 (2014)

6. W.M. Jacobs, A. Reinhardt, D. Frenkel, Proc. Nat. Acad. Sci. USA 112, 6313 (2015)

7. F.J. Martinez-Veracoechea, D. Frenkel, Proc. Nat. Acad. Sci. USA 108, 10963 (2011)

8. C.T. Varner, T. Rosen, J.T. Martin, R.S. Kane, Biomacromolecules 16, 43 (2015)

9. C.A. Mirkin, R.L. Letsinger, R.C. Mucic, J.J. Storhoff, Nature 382, 607 (1996)

10. P.L. Biancaniello, A.J. Kim, J.C. Crocker, Phys. Rev. Lett. 94, 058302 (2005)

11. N. Geerts, E. Eiser, Soft Matter 6, 4647 (2010)

12. P. Varilly, S. Angioletti-Uberti, B.M. Mognetti, D. Frenkel, J. Chem. Phys. 137, 094108 (2012)

13. L.D. Michele, E. Eiser, Phys. Chem. Chem. Phys. 15, 3115 (2013)

14. B.D. Myers, Q.Y. Lin, H. Wu, E. Luijten, C.A. Mirkin, V.P. Dravid, ACS Nano, Article ASAP, doi: 10.1021/acsnano.6b02246 (2016) 
15. C.S. Mahon, D.A. Fulton, Nat. Chem. 6, 665 (2014)

16. R. de laRica, R.M. Fratila, A. Szarpak, J. Huskens, A.H. Velders, Angew. Chem. Inter. Ed. 50, 5704 (2011)

17. N.B. Tito, S. Angioletti-Uberti, D. Frenkel, J. Chem. Phys. 144, 161101 (2016)

18. R.J. Rubin, J. Chem. Phys. 43, 2392 (1965)

19. S. Hong, P.R. Leroueil, I.J. Majoros, B.G. Orr, J.R. Baker Jr., M.M. Banaszak Holl, Chem. \& Biol. 14, 107 (2007)

20. N.A. Licata, A.V. Tkachenko, Phys. Rev. Lett. 100, 158102 (2008)

21. C. Nicosia, J. Huskens, Mater. Horiz. 1, 32 (2014)

22. Y. Li, Y.D. Tseng, S.Y. Kwon, L. d'Espaux, J.S. Bunch, P.L. McEuen, D. Luo, Nat. Mater. 3, 38 (2003)

Open Access This is an Open Access article distributed under the terms of the Creative Commons Attribution License (http://creativecommons.org/licenses/by/4.0), which permits unrestricted use, distribution, and reproduction in any medium, provided the original work is properly cited. 